\title{
REVOHPR: Relay-based Void Hole Prevention and Repair by Virtual Routing in Clustered Multi-AUV Underwater Wireless Sensor Network
}

\author{
Amir Chaaf \\ School of Communication and \\ Information Engineering, Chongqing \\ University of Posts and \\ Telecommunications, Chongqing, \\ China. \\ chaaf.amir@gmail.com
}

\author{
Mohammed Saleh Ali Muthanna \\ School of Computer Science and \\ Technology, Chongqing University of \\ Posts and Telecommunications, \\ Chongqing, China, \\ muthanna@mail.ru
}

Soha Alhelaly

College of Computing and Informatics, Saudi Electronic University, Riyadh, Saudi Arabia, s.alhelaly@seu.edu.sa
Ibrahim A. Elgendy

School of Computer Science and Technology, Harbin Institute of Technology, Harbin, China. ibrahim.elgendy@hit.edu.cn

\author{
Ahmed A. Abd El-Latif \\ Mathematics and Computer Science \\ Department, Faculty of Science, \\ Menoufia University, Shebin El-Koom, \\ Egypt. \\ a.rahiem@gmail.com
}

\author{
Ammar Muthanna \\ Department of Telecommunication \\ Networks and Data Transmission, The \\ Bonch-Bruevich Saint-Petersburg State \\ University of telecommunications, \\ 193232 Saint Petersburg, Russia, \\ Department of Applied Probability \\ and Informatics, Peoples' Friendship \\ University of Russia (RUDN \\ University) 6 Miklukho-Maklaya St, \\ Moscow, 117198, Russian Federation, \\ muthanna.asa@spbgut.ru \\ Abdullah M. Iliyasu \\ Electrical Engineering Department, \\ Prince Sattam Bin Abdulaziz \\ University, Al-Kharj 11942, Saudi \\ Arabia \\ School of Computing, Tokyo Institute of \\ Technology, Yokohama 226-8502, \\ Japan \\ School of Computer Science and \\ Technology, Changchun University of \\ Science and Technology, Changchun \\ 130022, China, \\ a.iliyasu@psau.edu.sa
}

\begin{abstract}
Underwater Wireless Sensor Networks (UWSN) enables various oceanic applications which require effective packet transmission. In this case, sparse node distribution, dynamic network topology and inappropriate selection of relay nodes cause void holes. Addressing this problem, we present a Relay based Void Hole Prevention and Repair protocol (ReVOHPR) by multiple Autonomous Underwater Vehicles (AUV) for UWSN. ReVOHPR efficiently identifies and avoids void holes and trap relay nodes to avoid it. ReVOHPR adopts the following operations as Ocean Depth (levels) based Equal Cluster Formation, Dynamic Sleep Scheduling, Virtual Graph based Routing, and Relay Assisted Void Hole Repair. For energy efficient cluster forming, Entropy based Eligibility Ranking (E2R) is presented which elects stable cluster heads (CHs). Then, dynamic sleep scheduling is implemented Dynamic Kernel Kalman Filter (DK2F) algorithm in which Sleep and Active modes based on the nodes current status. Inter Cluster Routing is performed by maximum matching nodes which selects by Dual criteria and also data transmitted to AUV. Finally, void holes are detected and repair by BiCriteria Mayfly Optimization (BiCMO) algorithm. The BiCMO focuses on reducing the number of holes, data packet loss and maximizes Quality of Service $(Q 0 S)$ and energy efficiency of the networks. This protocol is timely deal with node failures in packet transmission via multi-hop routing.
\end{abstract}

Simulation is implemented by NS3 (AquaSim module) simulator that evaluates the performance in network simulation for following metrics as average energy consumption, delay, packet delivery rate and throughput.

Keywords-Underwater Wireless Sensor Network, Void Hole Prevention and Repair, Clustering, Virtual Routing, Multi-AUVs and Relay Selection

\section{INTRODUCTION}

Underwater Wireless Sensor Network (UWSN) has many applications over the ocean environment. In UWSN, energy efficiency is the major constraint since the nodes are resource constraint [1], [2], [3]. To achieve energy efficiency, various approaches were presented in UWSN. Here, the data transmission is carried over multiple hops and further collision free medium access (MAC) protocols were presented. However, routing is also the best way to improve energy efficiency [4]. A cluster-based mobile data gathering is used to improve energy efficiency in the large-scale network. The basic cluster concept is considered in this work to form initial clusters. This cluster formation is performed in non-optima manner which is inefficient. 
However, Cluster Head $(\mathrm{CH})$ is performed in random manner which makes this work ineffectual [5]. In addition, processing distributed clustering algorithm needs large number of control packet exchange which consumes lots of energy. Autonomous Unmanned Vehicles (AUVs) are specially designed for data gathering in underwater environment [6], [7]. An AUV-assisted energy efficient clustering UWSN mechanisms faces many serious issues as [8], [9],

- Energy consumption in existing research works is high which leads to large number of holes in the network

- Network clusters with unequal size introduces energy imbalance in certain regions

- Optimal sleep scheduling is necessary in order to avoid holes

- Route selection considers only limited metrics which leads to large packet loss and energy consumption

In AUV assisted UWSN, the predefined path determination is the critical issue which increases the energy consumption and delay in data transmission [10]. [11]. On the other hand, the unnecessary sensing of the sensor nodes increases the energy consumption. These are only limited since the forwarder selection mechanism must consider more criteria. Further, route selection based on single metric is ineffective in underwater scenarios [12], [13]. Traditional routing algorithms follows ocean depth based routing. This lead to high packet loss due to void hole issue. Void hole avoidance is an emerging part of UWSN. Further, it is not able to avoid in the sparse node distribution with limited amount of energy. In addition, various important issues remain untouched in UWSN for reducing the energy consumption [14], [15].

- There is no unified protocol for reliable and energyefficient data transmission for specific type of UWSN

- Existing protocols focus on one aspect for energy consumption i.e. clustering, routing or void hole repair. Hence, energy consumption may occur by other aspects of issue.

- Current protocols used single AUV for data collection, which increases end-to-end delay of each sensor and thus energy consumption rate is increased [16], [17].

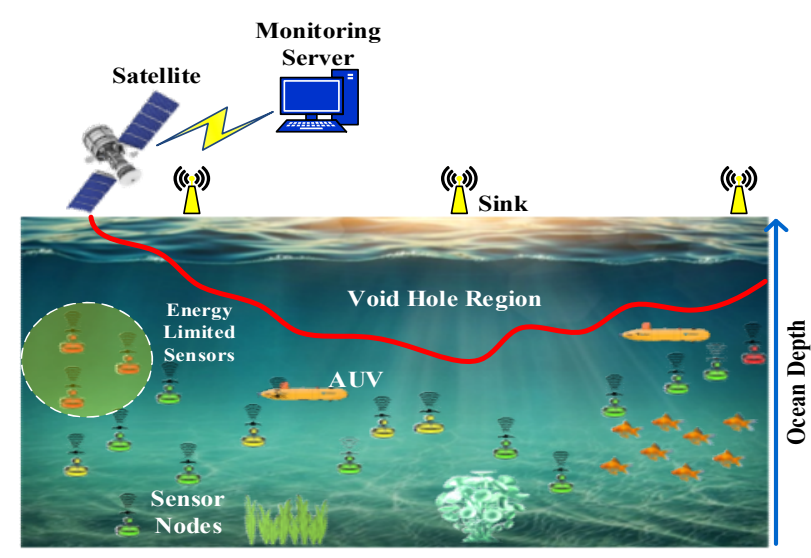

Fig.1. Void Hole Problem in UWSN

\section{A. Motivation}

Fig 1 illustrates the void hole problem in UWSN. In UWSN, presence of routing void holes leads to higher packet loss which makes the data unreliable. The main cause for routing voids is the higher energy consumption of the sensor nodes in the network (i.e.) nodes which losses energy makes the hole. In this context, there are two major research problem arises [18], [19], [20], [35].

- Most of the works have concentrated on energy efficient route selection without deploying AUV in the network. In this case, the energy consumption and delay for data transmission is high. Although these works select optimal route, it fails to transmit the data in timely manner since the hole mitigation process is generally transmits the data in longest path or backward path.

- In some works, AUVs are deployed to mitigate the problem of void holes. However, there is issue in predicting the trajectory of the AUVs since the travel length is high. As the trajectory detection methods uses the energy level alone for optimal positioning.

- The network is managed with unequal clusters which imbalances the load among clusters. Thus, some of the cluster heads suffer with higher energy consumption while some cluster heads suffer with lower energy consumption. In general, all underwater sensor nodes are continuously senses the environment which consumes lot of energy and introduces void holes. In the absence of optimal sleep scheduling mechanism, the energy consumption will be high and the holes are unavoidable.

This paper mainly focuses on void hole prevention and mitigation in underwater wireless sensor networks (UWSNs). For that, we intend to perform relay assisted void mitigation mechanism. We also prevent the holes by minimizing overall energy consumption in the network. This research work is motivated from the problems presented in the existing research works.

\section{B. Contributions}

In this paper, we deeply tackle all the issues of reducing the energy consumption in UWSN. In particular, we presented the following contributions to address the energy consumption and QoS issues.

- Level based Equal Clustering Algorithm (LECA) is presented that utilizes distance and load criteria. In each level, equal clusters are formed to avoid energy consumption. Entropy based Eligibility Ranking (E2R) protocol is presented for $\mathrm{CH}$ selection. Optimal $\mathrm{CH}$ is selected in two levels based on energy, centrality and success rate which increase the lifetime of $\mathrm{CH}$.

- To reducing energy consumption, dynamic sleep scheduling is presented by dynamic kernel based kalman filter (DK2F) is proposed. This considers Residual Energy, Buffer Value, Coverage Rate to make the decision on node status (Sleep / Active).

- Optimal route is selected in multiple hops by considering multiple factors. A virtual graph based routing is presented which uses Maximum Matching Theory for optimum selection of next hops. Optimal positioning of AUV repairs the voids in the network. 
Proposed Maximum matching approach choses optimal criteria according to the position of AUV.

- The optimal repair position of AUV is computed by Mayfly optimization algorithm is proposed and works upon multiple criteria to find the optimal trajectory of AUV. Mayfly algorithm works well in terms of convergence rate and convergence time

- Overall network follows level based clustering and optimal routing which minimizes the energy consumption that prevents the holes. Sleep scheduling prevents the nodes to dead, which further prevents the hole.

\section{Paper Layout}

The remaining part of the paper is organized as follows: Section II presents the literature review in the area of clustering, routing, sleep scheduling, and void hole detection and repair in UWSN. Section III focuses on the problems that are primarily existed in void hole avoidance. Section IV describes the research methodology, pseudocode and algorithms in detail. Section V illustrates the performance of network simulation for the proposed and previous protocols. Section VI concludes the paper and presented the future works.

\section{LiETARTURE REVIEW}

An energy efficient data gathering (EEDG) scheme was proposed in [21] for underwater wireless sensor network. The data transmission is performed in a multi-hop manner. At first, the energy consumption is balanced by grouping the nodes into smaller groups. Further, the forwarder nodes are selected to gather the data from subset nodes. Here, communication is carried in one-hop manner. Further, medium access control (MAC) protocol is utilized to improve collision rate and packet loss.

A fault resilient routing for underwater wireless sensor network was presented in [22] for underwater data transmission. The fault tolerant routing follows moth flame optimization (MFO) algorithm. The data transmission is carried through AUVs to base stations. Here, the AUVs act cluster heads that are responsible to collect data from the sensor nodes. Use of AUVs avoids re-clustering and overloading problem. To overcome the path disjoint issues, additional mobile nodes are deployed in the network. In this work, multiple AUVs are deployed to support data forwarding in the underwater network.

A cluster-based mobile data gathering (CMDG) scheme was studied in [23] for large-scale underwater sensor network. At first, the cluster formation and $\mathrm{CH}$ selection problem is formulated as optimization problem. In this work, the AUV tour planning scheme is presented to handle the sensor mobility. In order to achieve energy-latency tradeoff, the travel length is shortening for AUV movement. A centralized clustering algorithm is proposed to form initial clusters. Then, the distributed clustering algorithm is proposed to maintain the formed clusters. This work has two drawbacks as (1). $\mathrm{CH}$ Selection is inefficient since it considers only minimum number of parameters, and (2). Distributed clustering algorithm exchanges large number of control packets which is ineffective.
AUV assisted energy efficient clustering (AEC) mechanism was presented in [24]. The proposed AEC mechanism introduces wake-up sleep cycle for the underwater sensor network. The overall mechanism includes cluster formation, cluster head nomination and sleep wakeup scheduling. To form clusters, virtual sectoring approach is presented. In each virtual sector, cluster is formed and $\mathrm{CH}$ is selected. The $\mathrm{CH}$ is selected based on the distance with the cluster centroid point. Then, the path of AUV is predefined path. The predefined path of AUV is inefficient since it consumes large amount of energy and increases delay.

Author proposes a cluster based sleep scheduling mechanism in UWSN [25]. The overall network is considered as 3D underwater sensor network. A 3D partition unit is considered with a basic cluster structure. All sensor nodes are in the temporary control of clusters. In each cluster, sleep-awake scheduling is enabled based on the remaining energy level. The major goal of this work is to achieve minimum energy consumption and guarantee maximum sensing coverage in the network. Cluster formation in performed in non-optimal manner which is inefficient.

In [26] authors proposes a two-stage routing protocol. The main of this protocol is to enable communication between not only connected nodes but also for non-connected or partially connected nodes so that the packet delivery rate will be improved. To delay the death of nodes, an energy threshold method and rerouting scheme is proposed. Involvement of energy threshold and rerouting processes improve the connectivity of the network which prevents the holes. To preserve resource constraints, forwarding loops. However, unnecessary sensing of the sensor nodes increases the energy consumption.

An energy balanced efficient and reliable routing (EBER2) protocol for UWSNs was presented in [27]. Energy balancing among neighbors and reliability are achieved in EBERR protocol. The EBERR protocol considers residual energy and potential forwarding nodes (PFNs) of the forwarder node. The transmission range is divided into power levels and the forwarders can adjust the transmission range adaptively. In order to suppress the duplicate packets, depth of the nodes is compared. Forwarder selection is inefficient since it considers only limited metrics.

A distance vector based opportunistic routing (DVOR) protocol was proposed to address the problem of void regions in underwater sensor network [28]. The main idea behind this protocol is to use the depth information for route selection. The DVOR uses a query based mechanism to enable the distance vectors for underwater sensor networks. From the distance vectors, each node records the smallest hop count information towards sink node. Based on this hop count information, routing is performed. The void hole is avoided by selecting a route with small distance. When the network is sparse, then the hop count information will have large distance which increases the energy consumption. Optimal route selection based on single metric is insufficient to cope with UWSN.

In [29] sink mobility i.e. AUV and courier nodes are deployed in network for data collection, aggregation and transmission. The entire network is divided into four sectors. 
Both courier nodes and AUV are movable with random trajectory. In this protocol, routing is fixed and sink mobility is dynamic. Comparison is made between several existing protocols and the proposed protocol. However the mobility of mobile sink and courier nodes increases energy consumption and decrease the network lifetime. This is a linear type of network and it does not suited for complex ocean depth scenarios and also realistic applications are not adopted with this protocol. In [30], authors proposed a new data collection protocol for QoS provisioning. For that, bioinspired routing algorithm is proposed which facilitate the natural features of genetic algorithm. Clusters are formed which provides highly stable and different size of clusters for traffic load balancing. The proposed routing algorithm predicts high stable links as a forwarding node. This work eliminates the data transmission by the Upward and Downward transmission. The main drawback of this protocol is it does not aware of node mobility of packet delivery in UWSNs. In [31], fuzzy clustering is presented which designs the fitness function for selecting the $\mathrm{CH}$ by distance between the nodes. For cluster formation, fuzzy algorithm is used whereas $\mathrm{CHs}$ are selected by PSO algorithm. The overall network topology is arranged in a hierarchical structure and the comparative analysis is made between the proposed hybrid (Fuzzy and PSO) algorithm with LEACH and traditional PSO algorithms. This hybrid protocol has several drawbacks:

- Hybrid fuzzy and PSO algorithms consumes more energy of underwater sensors since computation of both algorithms is very high. Due to limited battery issue of underwater sensor, this protocol is not suited.

- The overall work partially reduces the energy level, which does not suit for risky oceanic applications and also it does not increase the lifespan of the UWSN.

In [32], void hole alleviation issue is addressed using enhanced geographic and opportunistic routing protocol in harsh underwater WSN. There are three problems such as Void Hole Occurrence, Higher Energy Consumption and Low Packet Delivery Rate are addressed in this paper. Further, network scalability issue is addressed in this paper. The performance of the proposed protocol is compared with the geographic and opportunistic routing with depth adjustment based topology control protocol and transmission adjusted neighbor node approaching distinct energy efficient mate's algorithms.

\section{PRoBlem STATEMENT}

The purpose of this paper is to investigate the problem of void hole and repair in multi-AUV enabled UWSN. This section summarizes the important issues in current works.

Authors in [34] focuses on void hole detection and mitigation in underwater sensor network. For that, two routing schemes are proposed. The first routing scheme namely energy-aware scalable reliable and void-hole mitigation routing (ESRVR) intends to avoid holes during route selection. In that, the two hop neighbor information is collected before initiation of route selection. As this scheme considers two hops the void hole is avoided in route selection itself. The second scheme namely cooperative ESRVR (Co-ESRVR) focuses on mitigating void holes through backward transmission. The major drawbacks of this paper are follows:

- Two-hop neighbor information alone is insufficient to avoid holes since the data from the nodes that are deployed in deep level needs to transmit through multiple hops. Thus there is a need for gathering multi-hop information which is not efficient.

- In the sparse network environment, it is hard to gather two-hop neighbor information. Since, it is not sure that always two-hop nodes will be presented.

- In backward routing energy consumption and delay is high. That is Co-ESRVR scheme also introduces multiple holes which need to be mitigated.

- When the network is sparse or there is limited possibility for backward transmission, then the ESRVR and Co-ESRVR are not feasible.

In [36] authors propose a game theoretic approach for energy efficient routing in 3D underwater WSN. However, game theory approaches have several drawbacks, which are follows.

- The game played by the nodes is non-cooperative. Thus, the strategy played by the players is unknown to other nodes. It leads to a same route is selected by multiple source nodes which introduces large number of collisions. Due to collisions packet retransmission count is high. This leads to large energy consumption.

- The game theory approach has high complexity. In addition, all players in the forwarding region is considered (other than neighbors) which makes the algorithm more complex.

Hence, high complexity in game theory approach requires more energy of UW sensors and also takes higher processing time. In [37] author proposes an AUV assisted data gathering approach to minimize energy consumption in Smart Ocean. For that, an AUV assisted data gathering scheme based on Clustering and Matrix Completion (ACMC) method for UWSN. The drawbacks in this work are follows.

- K-means algorithm forms clusters based on distance value. The formed unequal clusters will have imbalanced load among clusters. Presence of imbalanced load leads to energy consumption in some regions.

- $\mathrm{CH}$ and secondary $\mathrm{CH}$ are selected in each cluster. Here, the cluster center criteria are considered for $\mathrm{CH}$ selection while secondary $\mathrm{CH}$ is selected in a random manner. Thus, $\mathrm{CH}$ selection inefficient as the selected $\mathrm{CH}$ may lose the energy which becomes void holes.

- The greedy algorithm based AUV trajectory only considers the trajectory length. Means that nearby position is selected as AUV moving position. It doesn't make decisions based on the energy level of the nodes. If the nodes with low energy are located far away from the current AUV position, then there will be higher energy consumption. The greedy algorithm has higher time consumption and complexity

In [38] authors proposed an AUV assisted void prediction and repair mechanism in underwater sensor network. The repair position is calculated by Particle Swarm Optimization 
(PSO) algorithm. In this paper, PSO based void prediction causes more issues that are listed in follows.

- Repair position for AUV is computed by PSO which traps the solutions into local optima. It leads to nonoptimal positioning of AUV.

- Here, the single AUV collects the repair requests from multiple sensor nodes. And the rules are predefined. When the number of holes in the network is large, then the AUV suffers in decision making. The AUV couldn't handle the large amount of requests from the sensor nodes. Thus, this work is unable to mitigate the void holes effectively.

- This work only mitigates the void but unable to prevent the voids due to lack of optimal clustering and route selection procedures. The main reason for holes is high energy consumption. In this work, energy consumption is high in data collection, data transmission and sensing.

\section{SYSTEM MODEL}

\section{A. System Overview}

In this work, we present an energy efficient relay-assisted 3D-UWSN model in which void hole prevention and

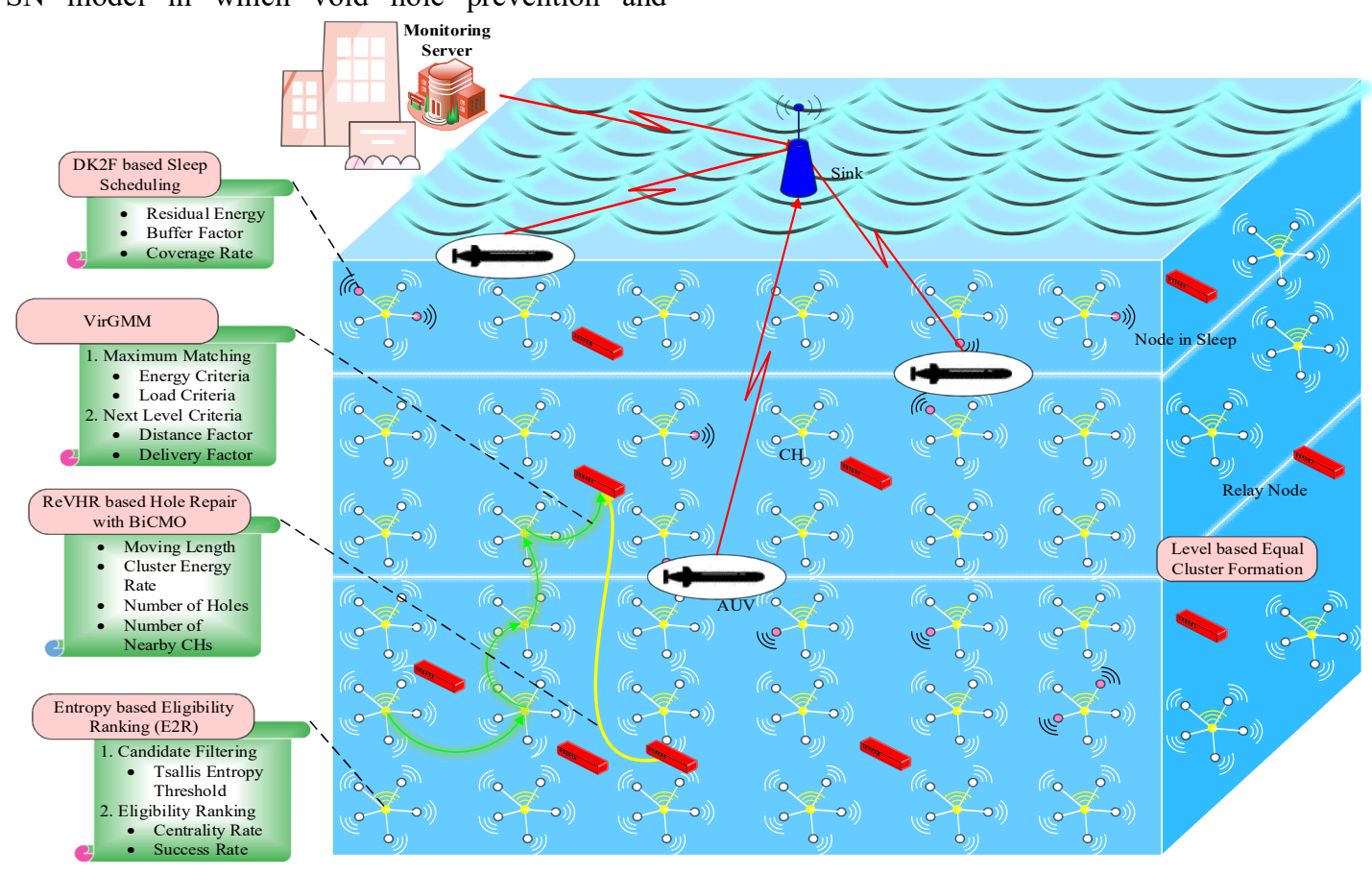

Fig.2. Network Model

The overall process comprises four major phases that are explained in the following sections. Fig 2 describes the overall network model. mitigation procedure is focused. The overall 3D-UWSN model comprises Underwater Sensor Nodes, Sink Node, Mobile Relays and Multiple AUVs. We construct the network based on multiple levels. In each level, an AUV is deployed to gather data from the underwater sensor nodes. All the nodes have the same initial energy $E_{\text {Ini }}$ and the sensing range $R_{S}$. Sensors can be transmitted into two types as Topology Information and Event. The size of the event and topology information packages is $\mathrm{M}$. The network connectivity rate is defined as the ratio of $S_{C}$ which is computed by $N(S)_{C}$ (number of sensors that communicates with the sink node) via single hop and multi-Hop communication which is computed as follows.

$$
S_{C}=\frac{N(S)_{C}}{n}
$$

When the network connectivity is 1 , then the network can obtain the full network connectivity and all sensors can communicate with the surface sink with either one hop or multi-hop communication. 


\section{B. Level based Equal Cluster Formation}

The overall network is segregated into multiple equal clusters based on the depth of the underwater environment. We propose a new Level based Equal Clustering Algorithm (LECA). In general, the nodes presented in underwater based sensor networks are resource constrained (i.e.) battery powered nodes. When energy level of a node is drained then that node is not able to participate in the network. So that the selected node must has enough energy to transmit the data. Thus E2R considers residual energy of a node as another metric. If any sensor has large amount of residual energy then that node has high possibility to become $\mathrm{CH}$.

\section{Energy Consumption Model}

Energy consumption in UWSN is focused by many research works since all sensor nodes involved in the network are energy constraint (i.e.) the nodes have energy sources that drops according to usage. Higher energy consumption leads to nodes early dead which minimizes network lifetime. The energy consumption of a node $n_{i}$ is formulated as follows,

$E\left(n_{i}\right)=E_{\text {idle }}\left(n_{i}\right)+\sum_{v \in V} \sum_{p \in P(v)} w(p) \times A(v) \times E\left(n_{i}, p\right)$

Assume a path $p \in P(v)$, then the weight of the path $w(p)$ is given as,

$$
\sum_{p \in P(v)} w(p)=1
$$

$A(v)$ is the average amount of energy consumed by node $n_{i}$ for a unit of time with respect to data transmissions, $E\left(n_{i}, p\right)$ is the amount of energy consumed only either in transmission or reception and $E_{\text {idle }}$ represents the average amount of energy consumed at idle state by the node $n_{i}$ for per unit time. From the estimated energy consumption of a node, the lifetime of a node is given as,

$$
\operatorname{LT}\left(n_{i}\right)=\frac{E_{\text {ini }}}{E\left(n_{i}\right)}
$$

Here, $E_{\text {ini }}$ represents the initial energy of the sensor node which is initially fed into the node for its participation in the network. The proposed LECA divides the network environment into multiple levels as $\mathrm{L}_{1}, \mathrm{~L}_{2}, \ldots, \mathrm{L}_{\mathrm{n}}$. In each level, clusters are formed based on the load level. Then, each cluster performs Entropy based Eligibility Ranking (E2R) based $\mathrm{CH}$ selection protocol to select optimal $\mathrm{CH}$. Here, Tsallis entropy is utilized to formulate optimal energy threshold. Upon threshold value, candidate nodes are filtered by E2R protocol. The proposed E2R protocol uses Residual Energy Level $\mathfrak{R}_{\xi}$, Centrality Factor $\mathrm{C}_{\mathrm{f}}$ and Success Rate criteria $\mathrm{SR}_{\mathrm{c}}$.

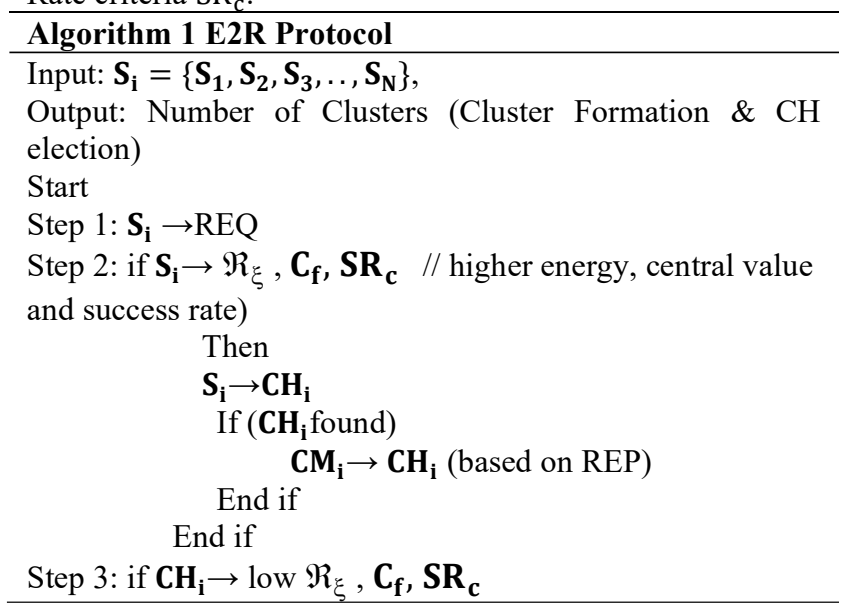

Then elect another $\mathrm{CH}$
\[ \begin{array}{l}\mathbf{C H}_{\mathbf{i}} \rightarrow \text { nearer } \mathbf{C H}_{\mathbf{i}} \\ \text { End if }\end{array} \]
Step 4: $\mathbf{C H}_{\mathbf{i}} \rightarrow$ SN
End

Algorithm.1 explains the procedure of E2R algorithm based cluster formation and $\mathrm{CH}$ election. Involvement of E2R algorithm improves the data aggregation process as well as network QoS performance. Further, involvement of E2R based data aggregation also helps to minimize the risk of stability in the network. Generalized Tsallis Entropy is computed by follows,

$$
T_{Q}\left(P_{1} \ldots P_{W}\right)=\frac{1}{1-q}\left(\sum_{i=1}^{w} p_{i}^{q}-1\right)
$$

Where $q$ is the logarithmic function. For all sensors in network, $T_{Q}\left(P_{1} \ldots P_{W}\right)$ is computed for the number of given input parameters. Using $T_{Q}$ the weight value for $\mathrm{CH}$ selection is implemented as,

$$
T_{Q}=\left(S_{1} \cdot C_{1}\right) w_{1} \times\left(S_{2} \cdot C_{2}\right) w_{2} \times\left(S_{3} . C_{3}\right) w_{3}
$$

Where $w_{1}, w_{2}, w_{3}$ are the weight values for $C_{1}, C_{2}$ and $C_{3}$, respectively.

The $\mathrm{CH}$ is selected based on,

$$
C H_{\text {prob }}=\max \left(C_{f_{\text {prob }}} \Re_{\xi_{\text {max }}}, S R_{c_{\max }}\right)
$$

After $\mathrm{CH}$ selection, cluster was formed based on sensor node's cost function as,

$$
C M(i, j)=\frac{S R_{c(i j)}}{S R_{c(\max )}} * \frac{E_{c u r}(i)}{E_{\text {cur }}(j)} * \frac{C(i j)}{C_{\text {ave }}}
$$

Where $E_{\text {cur }}(i)$ represent current energy of node i and $E_{\text {cur }}(j)$ represent current energy of $\mathrm{CH}$ j. Each $\mathrm{CH}$ in the cluster aggregates the sensor data and forward to the AUV. The proposed E2R protocol has $O(n)$ complexity which is due to the message transmission overhead where $n$ is the number of sensors. $\mathrm{CH}$ announce, join request, and join response messages are exchanged within the cluster, which introduce the complexity in cluster.

\section{Dynamic Sleep Scheduling}

In each cluster, dynamic sleep scheduling procedure is established in order to avoid unnecessary energy consumption. For that, we propose Dynamic Kernel Kalman Filter (DK2F) algorithm. In particular, Cauchy Kernel Function is used for non-linear cases. The DK2F algorithm takes Residual Energy Level $\mathfrak{R}_{\xi}$, Buffer Factor $\varsigma_{f}$, and Coverage Rate $\chi_{r}$ to make decision on the node status. The considered statuses are Sleep, Active and Transmit. The DK2F is executed by each node and the report is sent to the $\mathrm{CH}$. Then, the $\mathrm{CH}$ activates the mode for each node in the cluster. The procedure for DK2F is describes as follows. Firstly, kernel function is initiated which defined by,

$$
\delta_{\aleph}(e)=\frac{1}{1+e^{2} / \aleph}
$$

Where $e$ is the exponential term between two different variables and $S$ is the dynamic kernel bandwidth and $\delta$ range between 0 and $\infty$. DK2F gives the optimum solution for both linear and non-linear cases of the model in dynamic nature. The mathematical formulation of DK2F is follows.

$$
\begin{gathered}
X_{T}=f_{T} X_{T}+A_{T} \\
Y_{T}=h_{T} X_{T}+B_{T}
\end{gathered}
$$

Where $X_{T}$ is the state vector and $Y_{T}$ is the observation measurements at time $T . f_{T}$ and $h_{T}$ represents the State 
Transition matrix and Observation matrix, respectively. $A_{T}$ and $B_{T}$ denotes the noise values in observation and Gaussian noise, respectively. In probabilistic model, it is represented as
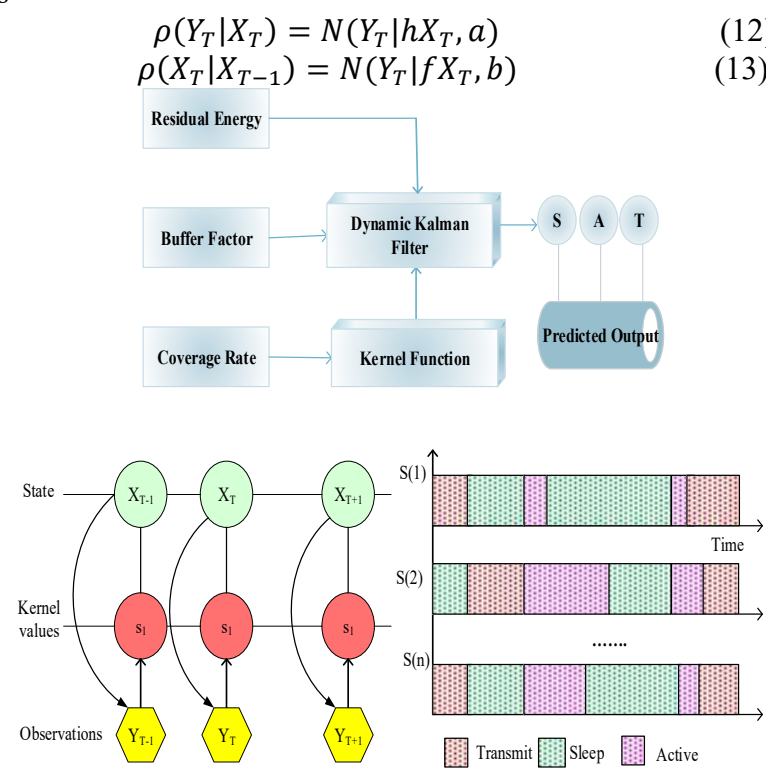

Fig 3. Dynamic Sleep Scheduling

Fig 3 illustrates the dynamic sleep scheduling model. The assumption and prediction of the underwater sensor node is illustrated as follows.

- Every node in underwater environment has follows only three kinds of states as Active, Sleep or Transmit. In active state, sensors are working that is listen the events and process the computations. Besides, it can also possible to switching to the idle state.

- All sensors can be possible to act as relaying state for packets transmission to the near AUV. For that each node maintains next hop nodes in the neighbors list.

- The duration of the active status is exponentially distributed with mean $1 / S$. In the active state, the sensors will sense packets, relay packets, and process packets. When all nodes are under sleep state, the $\mathrm{CH}$ cannot aggregate or transmit any packets. In this case, entire cluster puts into OFF state. When at least one node in active state, then $\mathrm{CH}$ turns into $\mathrm{ON}$ state again. In this case, $\mathrm{CH}$ can transmit or receive packets from cluster members. The actual and predicted result for the DK2F is indicated in fig 4.

\section{Pseudo code: member balanced scheduling}

Input: Total Cluster Members $i$

Output: Scheduled Mode

1. Begin

2. Initialize $i=\left\{s_{1}, s_{2}, s_{3}, \ldots.\right\} / *$ sensors in a cluster*/

3. For each $i$

3. Find $\Re_{\xi}, \varsigma_{f}$, and $\chi_{r}$

4. List $R E \varsigma_{f}$, and $\chi_{r}$ for each $k$

5. Find Dynamic Kernel Values

5. if $\left(\Re_{\xi}<\Re_{\xi_{T h}} \& \& \varsigma_{f}<\varsigma_{f_{(T h)}} \& \& \chi_{r}<\varsigma_{f_{(T h)}}\right)$

/*comparison with threshold*/

\{

assign sleep mode else

$$
\text { assign active mode }
$$

\}

end if

6. End for

7. End

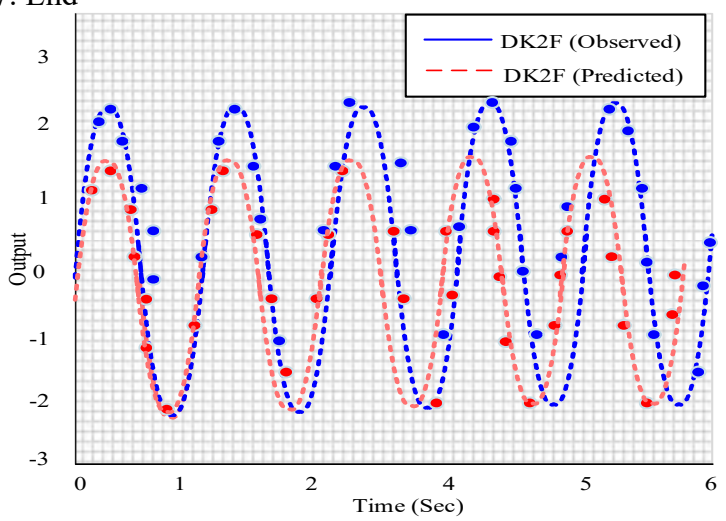

Fig.4. Performance Plot for DK2F

\section{Virtual Graph based Routing}

Inter-cluster routing is performed to improve energy efficiency and delivery rate. We present a novel Virtual Graph enabled Maximum Matching (VirGMM) algorithm. In first step, the virtual graph is constructed for the CHs. Then, Maximum Matching nodes are selected based on Dual-Criteria as Energy Criteria \{Residual Energy Level \& Expected Energy Consumption $\}$ and Load Criteria \{Current Load Level \& Expected Load Level\}. Among maximum matching nodes, optimal forwarder is selected upon optimum criteria. The criteria is selected upon the following rules,

\section{\{If AUV is presented in Same Level, then Choose [Delivery Rate Criteria]; Else, Choose [Distance Criteria]\}}

In this way, data is transmitted to AUV through optimal forwarders. Fig 5 describes the virtual graph based routing.

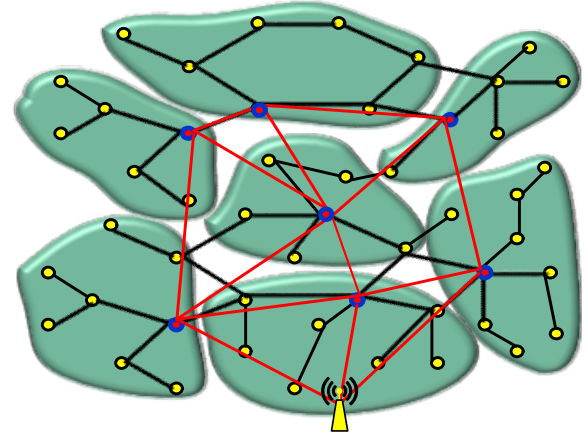

Fig.5.Virtual Graph based Routing

To minimize the sensors energy usage in data transmission, virtual graph with maximum matching theory is applied in which nearest next hop is selected for fast data transmission without any packet loss. Sensors in active state is sense the event about the environment and then send the sensed report to the sink node through AUV and next hops.

In this algorithm, bi-criteria is used such as $\mathfrak{R}_{\xi}$ and $E \mathfrak{R}_{\xi}$ for first criteria and $\mathrm{C}_{\mathrm{L}}, E \mathrm{C}_{\mathrm{L}}$ are considered as the second criteria to find the perfect match. For each node, the 
connectivity to become maximum match $(M M)$ is derived from Bayesian Theory as follows,

$$
\begin{gathered}
C\left(S_{i}\left(\Re_{\xi}\right) \mid M M\right)=\frac{C\left(M M \mid S_{i}\left(\Re_{\xi}\right)\right) C\left(S_{i}\left(\Re_{\xi}\right)\right)}{C(M M)} \\
C\left(S_{i}\left(E \Re_{\xi}\right) \mid M M\right)=\frac{C\left(M M \mid S_{i}\left(E \Re_{\xi}\right)\right) C\left(S_{i}\left(E \Re_{\xi}\right)\right)}{C(M M)} \\
C\left(S_{i}\left(\mathrm{C}_{\mathrm{L}}\right) \mid M M\right)=\frac{C\left(M M \mid S_{i} \mathrm{C}_{\mathrm{L}}\right) C\left(S_{i}\left(\mathrm{C}_{\mathrm{L}}\right)\right.}{C(M M)} \\
C\left(S_{i}\left(\mathrm{EC}_{\mathrm{L}}\right) \mid M M\right)=\frac{C\left(M M \mid S_{i} \mathrm{EC}\right) C\left(S_{i}\left(\mathrm{EC}_{\mathrm{L}}\right)\right.}{C(M M)}
\end{gathered}
$$

Where Eqn. (14) computes the probability of a node $S_{i}$ to become a next hop based on its $\Re_{\xi}$. Similarly, Eqn. (15) Eqn. (16) and Eqn. (17) compute the probability based on current load and expected load respectively. Here, the maximum matched values are mapped between 0 and 1 range. The source $\mathrm{CH}$ finds the $M M$ for all available next hops and the sorted the best set of matches. The set of possible matches by matching theory is illustrated in table.1

Table.1 Set of Rules for Forwarder Selection

\begin{tabular}{|c|c|c|c|c|}
\hline $\mathfrak{R}_{\xi}$ & $E \Re_{\xi}$ & $\mathrm{C}_{\mathrm{L}}$ & $\mathrm{EC}_{\mathrm{L}}$ & $\begin{array}{c}\text { Status of } \\
\text { node }\end{array}$ \\
\hline$<0.5$ & $<0.5$ & $<0.5$ & $<0.5$ & $\begin{array}{c}\text { Partially } \\
\text { Match }\end{array}$ \\
\hline$<0.5$ & $<0.5$ & $>0.5$ & $>0.5$ & No Match \\
\hline$<0.5$ & $>0.5$ & $<0.5$ & $<0.5$ & No Match \\
\hline$<0.5$ & $>0.5$ & $>0.5$ & $>0.5$ & No Match \\
\hline$>\mathbf{0 . 5}$ & $<\mathbf{0 . 5}$ & $<\mathbf{0 . 5}$ & $<\mathbf{0 . 5}$ & Highly Match \\
\hline$>0.5$ & $<0.5$ & $>0.5$ & $>0.5$ & $\begin{array}{c}\text { Partially } \\
\text { Match }\end{array}$ \\
\hline$>0.5$ & $>0.5$ & $<0.5$ & $<0.5$ & $\begin{array}{c}\text { Partially } \\
\text { Match }\end{array}$ \\
\hline$>0.5$ & $>0.5$ & $>0.5$ & $>0.5$ & No Match \\
\hline
\end{tabular}

D. Relay-assisted Void Hole Repair

Routing void is detected by the neighboring nodes. Once the void hole is detected, then the report is generated and sent to the AUV. The AUV then takes optimal decision on the void hole repairing. In this, we introduced novel Relayassisted Void Hole Repair Mechanism (ReVHR). On receiving void requests, the AUV selects an optimal relay node to repair the hole. The relay node selection is carried based on Distance Factor. Then, optimal position of the relay is determined by Bi-Criteria Mayfly OREVOHPRptimization (BiCMO) algorithm. The BiCMO considers the following objective functions,

$$
O F=\left\{\operatorname{Min}\left[m_{l}\right] \& \& \operatorname{Min}\left[n_{(h)}\right]\right\}
$$

Where $m_{l}$ is the moving length and $n_{(h)}$ is the number of holes. The moving distance, Number of Holes, Number of $\mathrm{CHs}$, and Energy level of the region are considered to reposition the void holes. Once the relay node is repositioned, then the source $\mathrm{CH}$ transmits the data to AUV through relay node. In this way, the void hole is repaired and the data is transmitted to AUV without loss.

\begin{tabular}{l}
\hline Algorithm 2: May Fly based Relay Node Selection \\
\hline Input: $n$ number of nodes \\
Output: Void Repair \\
Procedure: \\
1. Initialize population \\
2. For all sensors \\
3. Fitness estimation \\
4. Determine total fitness ()$=(1),(2), \ldots \ldots$ \\
5. Calculate probability of each node \\
\hline
\end{tabular}

6. Identification of Relay Node

$$
\text { If (Probability }==\text { High) }
$$$$
\text { Assign Node } \rightarrow \text { VHR } \quad / / \text { VHR - Void }
$$$$
\text { Hole Repair }
$$$$
\text { Alert broadcasted to next relay nodes }
$$

Else

End if

Move to next node

8. End for

9. End

Algorithm 2 describes the pseudocode for Mayfly based replay selection. The fitness value is computed for each node by bi-objectives.

\section{Simulation Results}

In this section, the simulation results are presented for the proposed REVOHPR protocol is evaluated in terms of energy consumption, packet delivery ratio, and throughput for effective data transmission and hole detection and repair mechanisms. In addition, the proposed ReVHR protocol is compared to similar UWSN protocols such as ESRVR [34], ACMC [36], and PSO [32,38]. The detailed description of the simulation environment and comparative study are specified as follows.

\section{A. Simulation Setup}

The simulation of the proposed vs. existing protocols for data transmission in UWSN is implemented using NS3.27. In NS3, AquaSim is one of the significant modules for underwater sensor environment simulation and besides other modules are supported to create the network model. The simulation parameters that used in the proposed REVOHPR model are illustrated in Table.2. The simulation parameters are not constrained by any limit. The simulation is performed using Ubuntu 14.04 LTS operating system with 32 bit processor. When compared to other simulators, NS3

\begin{tabular}{|c|c|c|}
\hline \multicolumn{2}{|c|}{ Parameter } & Specification \\
\hline \multirow{7}{*}{$\begin{array}{c}\text { Network } \\
\text { Parameters }\end{array}$} & Simulation Area & $1000 \times 1000 \times 1000$ \\
\hline & $\begin{array}{c}\text { \# of Underwater } \\
\text { Sensors }\end{array}$ & 100 \\
\hline & \# of Relay Nodes & 10 \\
\hline & \# of AUVs & 3 \\
\hline & \# of Sink & 1 \\
\hline & Number of clusters & $5-7$ \\
\hline & Simulation Time & 300 Seconds \\
\hline & Modules Used & $\begin{array}{c}\text { AquaSim, } \\
\text { Antenna, Config } \\
\text { Store, CSMA, } \\
\text { LTE, AODV, } \\
\text { Mesh, Mobility, } \\
\text { DSR, Flow } \\
\text { Monitor, and } \\
\text { Internet } \\
\end{array}$ \\
\hline \multirow[b]{3}{*}{ Underwater } & Packet size & $512 \mathrm{~KB}$ \\
\hline & \# of packets & $20-200$ \\
\hline & Packet Time & 100 Milli \\
\hline
\end{tabular}
is more flexible tool to simulate Clustering, Sleep Scheduling, Inter Cluster Routing and Void Hole Detection $\&$ Repair mechanism. The procedure for simulation is depicted in fig. 6

Table 2 NS3 Simulation Parameters 


\begin{tabular}{|c|c|c|}
\hline \multirow{3}{*}{$\begin{array}{c}\text { sensor } \\
\text { parameters }\end{array}$} & Interval & Seconds \\
\cline { 2 - 3 } & Data Rate & $10-20 \mathrm{Mbps}$ \\
\cline { 2 - 3 } & $\begin{array}{c}\text { Initial Energy Per } \\
\text { Sensor }\end{array}$ & $70 \mathrm{~J}$ \\
\cline { 2 - 3 } & $\begin{array}{c}\text { Transmission } \\
\text { Range }\end{array}$ & $300 \mathrm{~m}$ \\
\hline
\end{tabular}

As discussed in above, the simulation result is indicated in fig. Based on the data collected from AUV is processed in onshore sink.

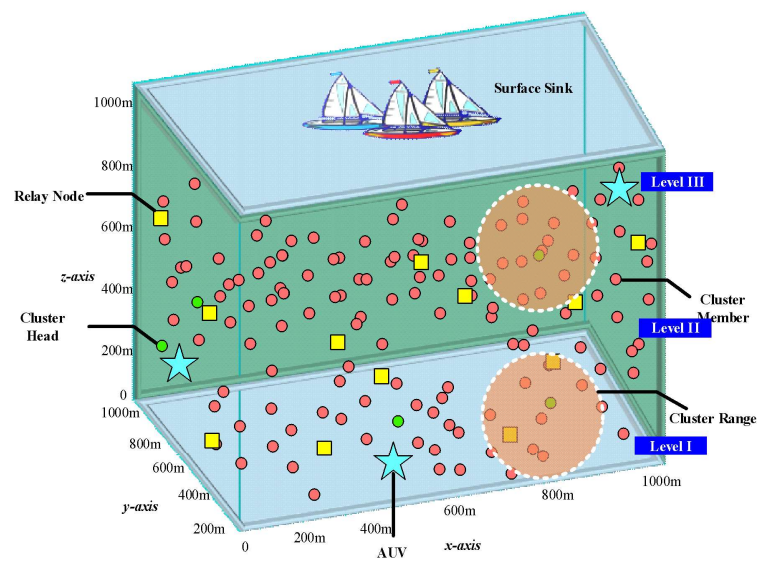

Fig.6.Simulation Setup for REVOHPR Protocol

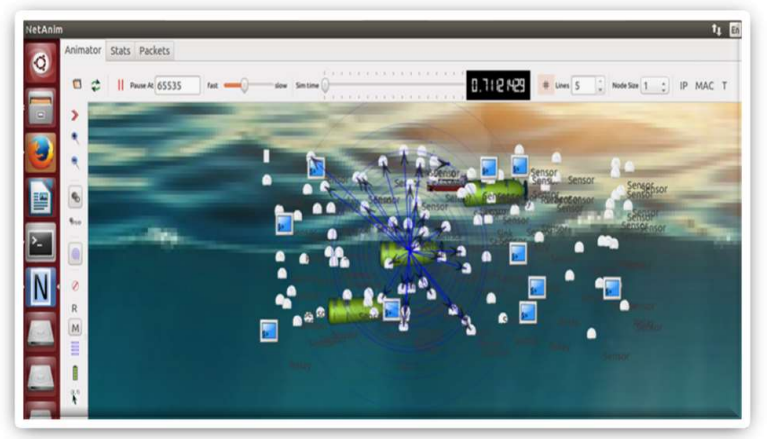

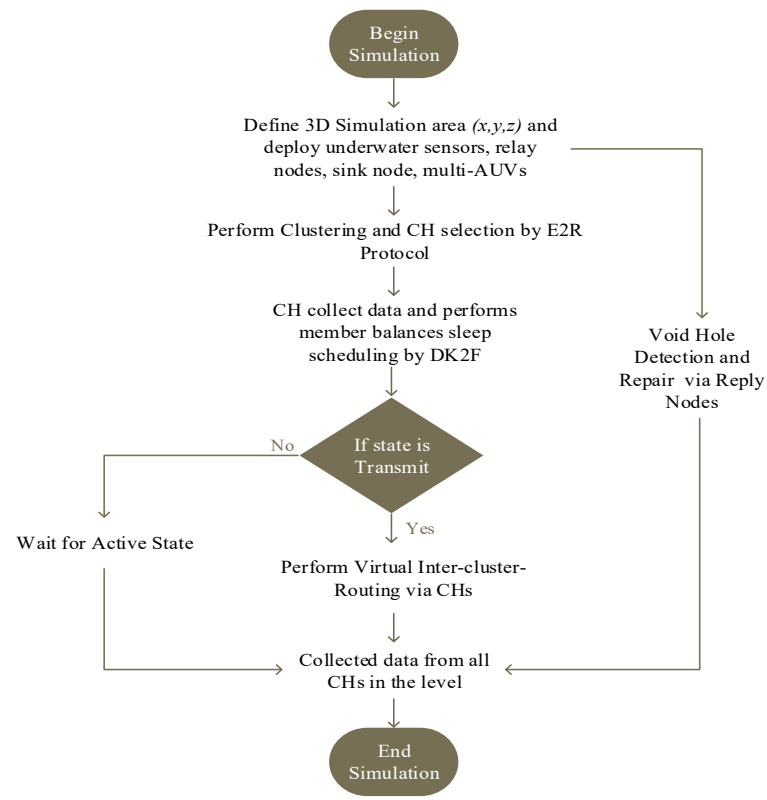

Fig.8.Flowchart for REVOHPR Protocol

Fig 8 shows the flowchart of the proposed ReVOHPR protocol. The performance of protocol is evaluated by this step. At the end of simulation, graphical plots are drawn by the simulation result. Our protocol is dynamic and supported for diverse nature (applications, and dynamic range of simulation).

Fig. 7.Simulation Running in NS3

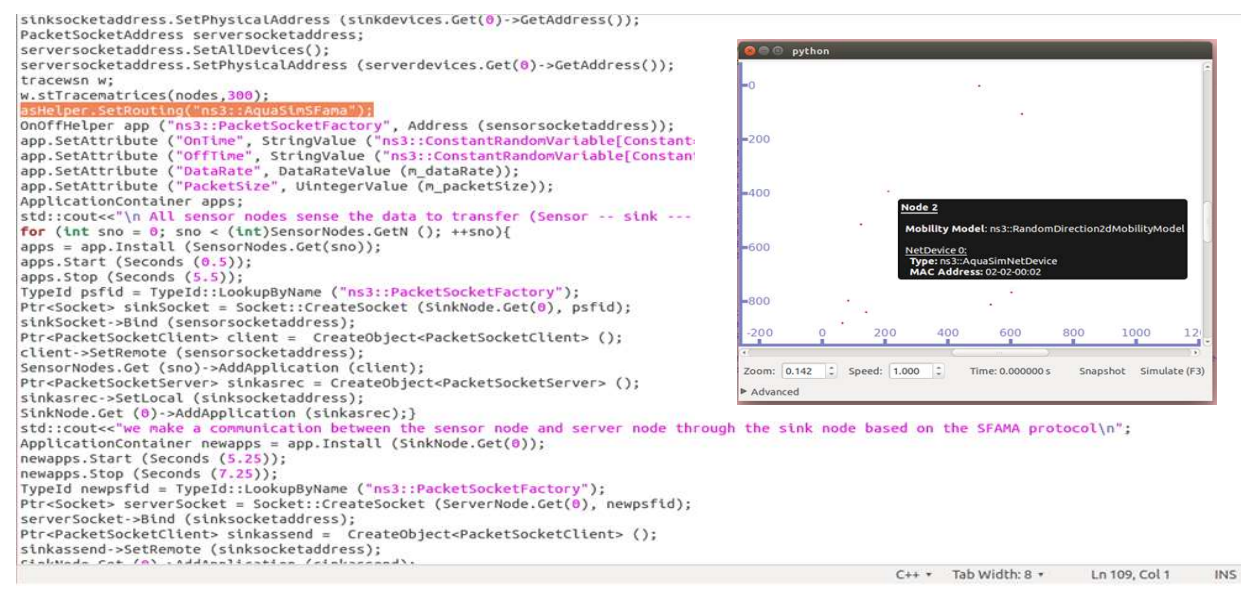

Fig.9.AquaSim Module in NS3 for Acoustic Communication

\section{B. Application Scenario: Sea Life Monitoring}

The proposed protocol is tested for sea life monitoring. In this case, sensors suitable for underwater animals monitoring is deployed in ocean and records real-time events from the environment. Two types of underwater sensors are used for sea life monitoring i.e. physical sensors and chemical sensors. Pressure, Oxygen, Temperature is the 
physical sensors whereas Salinity, turbidity, $\mathrm{PH}$, nitrate, chlorophyll, dissolved oxygen are the chemical sensors.

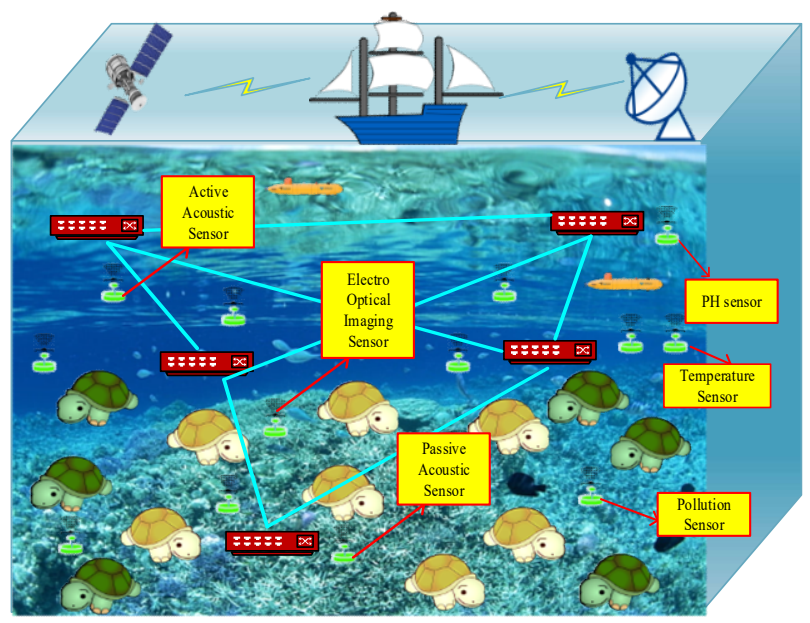

Fig.10.Sea Life Monitoring in UWSN

The representation of the sea life monitoring is depicted in fig 10. The type of sensors and their purpose is described in Table 3. The specification of each sensor is illustrated in table 4. Ocean climate is changed over a long period which is hazardous to the Marine Life. For example, abnormal sea temperatures affect the life of sea animals.

Table 3. Sea Life Sensors

\begin{tabular}{|c|c|c|}
\hline Animal Type & Animal Status & $\begin{array}{c}\text { Suitable Sea } \\
\text { Sensors }\end{array}$ \\
\hline Dolphins & Below Sea & Active Acoustic \\
Whales & Surface & Monitoring \\
Seals & & Sensors \\
Turtles & Frequently Near & Electro Optical \\
Fish & or at Sea Surface & Imaging Sensors \\
\cline { 2 - 3 } Porpoises & Frequent Distinct & Passive Acoustic \\
& Vocalization & Monitoring \\
& & Sensors \\
\hline
\end{tabular}

Table 4. Underwater Sea Life Monitoring Sensors

\begin{tabular}{|c|c|c|c|c|c|}
\hline Sensors & $\begin{array}{c}\text { Observed } \\
\text { pattern }\end{array}$ & Range & Accuracy & $\begin{array}{c}\text { Power } \\
\text { Supply }\end{array}$ & Unit \\
\hline $\begin{array}{c}\text { SBE16plus } \\
\text { V2 }\end{array}$ & Temperature & $\begin{array}{c}-5-+30 \\
{ }^{0} \mathrm{C}\end{array}$ & $\pm 0.0055^{0} \mathrm{C}$ & $\begin{array}{c}9- \\
30 \mathrm{~V}\end{array}$ & ${ }^{0} \mathrm{C}$ \\
\hline GT301 & Pressure & $0-60$ & $\begin{array}{c}< \pm 0.5 \% \text { of } \\
\text { FRO }\end{array}$ & $24 \mathrm{~V}$ & Bar \\
\hline $\begin{array}{c}\text { SBE16plus } \\
\text { V2 }\end{array}$ & Conductivity & $0-9$ & \pm 0.0005 & $\begin{array}{c}9- \\
30 \mathrm{~V}\end{array}$ & $\mathrm{~S} / \mathrm{m}$ \\
\hline $\begin{array}{c}\text { OBS }-3+ \\
\text { Turbidity }\end{array}$ & $\begin{array}{c}\text { Mud: } \\
5000- \\
10000 \\
\mathrm{mg} / \mathrm{L}\end{array}$ & $0.5 \mathrm{NTU}$ & $15 \mathrm{~V}$ & $\mathrm{NTU}$ \\
\hline PS 2102 & PH & $\begin{array}{c}0-14 \\
\mathrm{pH}\end{array}$ & \pm 0.1 & $\mathrm{~N} / \mathrm{A}$ & $\mathrm{pH}$ \\
\hline YSUS V3 & Chlorophyll & $\begin{array}{c}0- \\
400 \mu \mathrm{g} / \mathrm{L}\end{array}$ & $0.1 \mu \mathrm{g} / \mathrm{L}$ & $6 \mathrm{~V}$ & $\mu \mathrm{g} / \mathrm{L}$ \\
\hline SBE 63 & $\begin{array}{c}\text { Dissolved } \\
\text { Oxygen }\end{array}$ & $\begin{array}{c}120 \% \\
28 \mathrm{mg} / \mathrm{L}\end{array}$ & $\pm 0.028 \mathrm{mg}$ & $6-$ & $\mathrm{mg} / \mathrm{L}$ \\
& & 0.1 & $6-24 \mathrm{~V}$ & $\mathrm{mg} / \mathrm{L}$ \\
\hline
\end{tabular}

\section{Comparative Study}

In this section, we illustrate the performance analysis of the proposed and the previous protocols in terms of various QoS and energy consumption metrics as Energy Consumption, Delay, Throughput, and Packet Delivery
Ratio (PDR). The previous protocols for data transmission and void hole repair can be follows: ESRVR, ACMC and PSO.

\section{a. Energy Consumption}

Energy is a significant metric in underwater sensor communications. In underwater sensor network, acoustic signals transmitted in a cylindrical way. Higher energy consumption must be avoided since it represents the worst performance of the network. Packet transmission loss between two nodes is the major reason for higher energy consumption. This transmission loss $t(l)$ is computed by follows.

$$
t(l)=10 \log \frac{R_{1}}{R_{2}}
$$

Where $R_{1}$ and $R_{2}$ are the source and destination nodes of the transmission. Average energy consumption is the sum of energy consumed by all nodes in the network during Idle, Packet Transmission, Reception and Sensing. Hence, the average energy consumption is expressed by,

$$
\begin{aligned}
E_{(i)}= & \sum_{i=1}^{N} E\left(T x_{i}\right)+\sum_{i=1}^{N} E\left(R x_{i}\right)+\sum_{i=1}^{N} E\left(S e_{i}\right)+ \\
& \sum_{i=1}^{N} E\left(I_{i}\right)
\end{aligned}
$$

Where $N$ represents the total number of nodes, $T x_{i}$ is the sum of energy for transmission, $R x_{i}$ is the sum of energy for reception, $S e_{i}$ is the sum of energy consumed in sensing and $I_{i}$ is the node at idle state.

Fig 11 represents the sum of energy consumption rate of ReVOHPr against the previous protocols as ACMC, ESRVR, and PSO. Mathematical computations and number of iterations for cluster formation, $\mathrm{CH}$ election and routing considers more energy consumption. These processes were used to reduce the residual energy. The effective selection of $\mathrm{CH}$, and routing by virtual graph algorithm reduces overhead in packet transmission. Further, sleep scheduling idea is used which saves energy and improve the network lifetime. The graphical plots show that the amount of energy consumption is increases with respect to the number of nodes. However, network density is the primary factor that affects the network performance in terms of energy consumption and QoS. Fig 11 shows the performance of average energy consumption with respect to the number of packets per second. Data transmission is performed in multihop fashion. When the sensors in network communicate to the surface sink directly by Single Hop fashion, then the energy consumption rate is higher. Since single hop and multi-hop networks performance is not similar in energy consumption rate. Distance between the source to the sink is higher or lesser in single hop whereas multi-hop routing often has less number of relay nodes. In PSO and ESRVR, packets are transmitted in single hop to the surface sink. Compared to ACMC, ESRVR and PSO, the proposed ReVOHPR consumes minimum energy i.e. $37 \%, 42 \%, 67 \%$, respectively. 


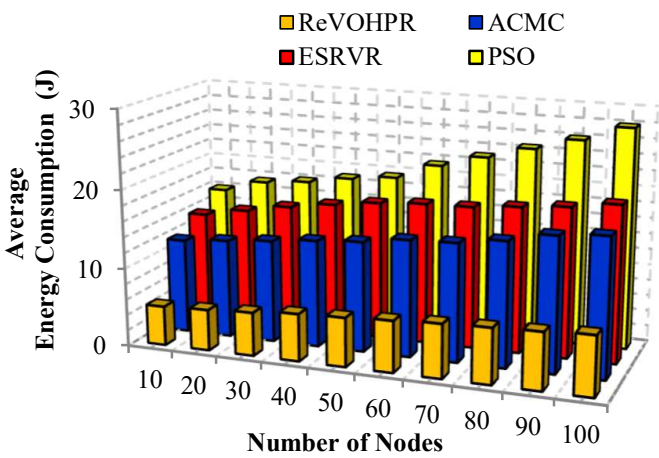

(a)

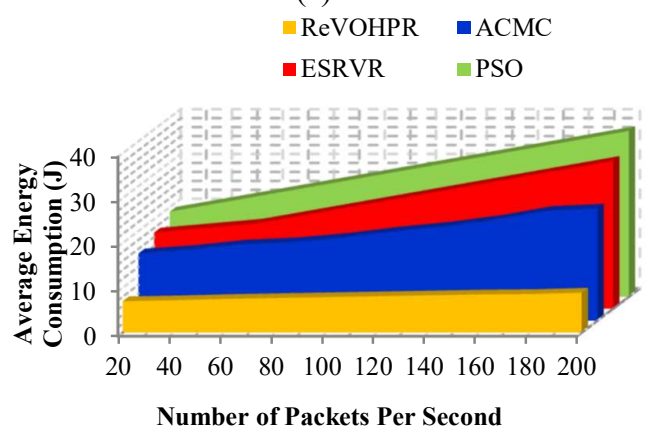

(b)

\section{b. Delay}

Fig.11.Impact of Energy Consumption

It is the amount of time required for data transmission from source (underwater sensor) $P_{(S)}$ to the destination node (surface) $P_{(D)}$. In other words, delay is computed from the generation to the destination reception. The successful packets are count up in delay computation. This computed by follows,

$$
D=P_{(S)}-P_{(D)}
$$

Fig 12 shows the performance of delay with respect to the number of nodes. The proposed ReVOHPR forwards packets by $\mathrm{CH}$ and aggregated packets forward to next hop using multi-hop routing algorithm. Ocean depth is the major element to consider in both clustering and routing. When the depth of ocean is higher, then the packet collection time is higher. The delay is higher in PSO when it process with packets without processing the void hole detection and prevention. The void management in the proposed ReVOHPR protocol improves the network data transmission and improves the nodes presence. Thus the delay in packet transmission is eliminated. PSO based algorithm is not sufficient for data transmission. Since, it is low convergence rate in the iterations.
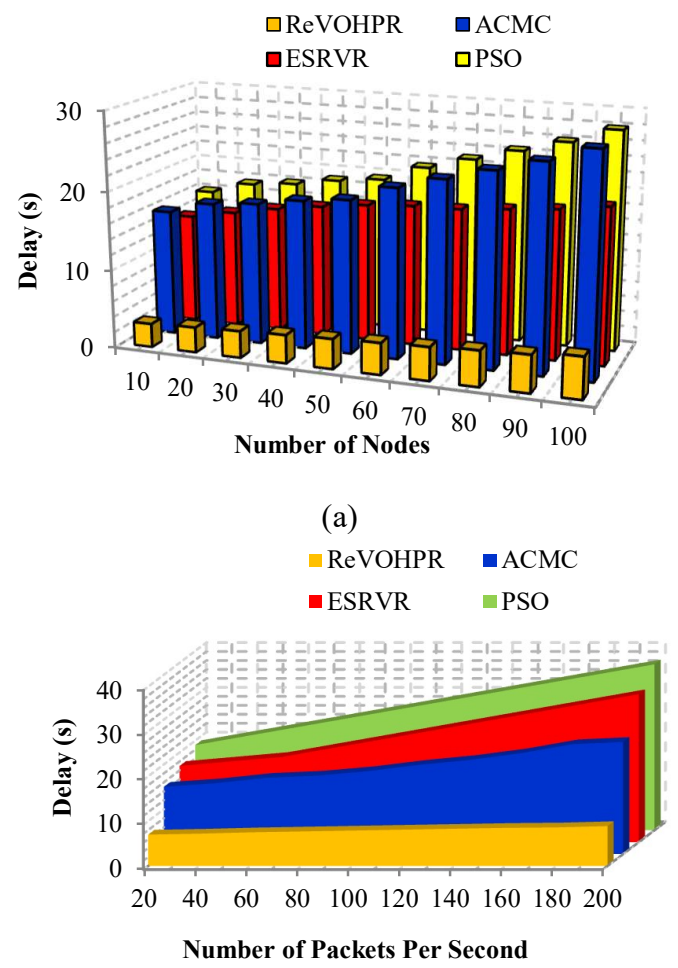

(b)

Fig.12.Impact of Delay

The total delay required for data transmission from source to the destination with respect to the number of packets is very higher for previous protocols. It is illustrated in fig 12. It is computed by several factors as propagation delay, transmission delay, number of hop counts, and distance between two nodes. ReVOHPR utilizes the relay based void hole prevention and repair. This efficiently helps to identify the presence of void and handles it precisely. The increased frequency of void occurrence increases delay when the number of nodes increases for lengthy route. The computational time required in high traffic congestion is exponential and it does not suited for events based data transmission.

\section{c. Throughput}

It is the positive metric that defines the amount of packet transmitted in a time. When measuring the maximum throughput in packet transmission, then the communication link or network access is reliable. It is computed by follows:

$$
\text { Throughput }\left(\frac{\text { bits }}{\text { sec }}\right)=\frac{\operatorname{Sum}(N(S p) * A P S)}{T(t)}
$$

Where $N(S p)$ is the number of successful packets, APS is the average packet size and $T(t)$ is the total time spend for packet transmission. The simulation results in fig 13 shows that the performance of the proposed ReVOHPR is higher than the previous protocols. It is analyzed by both number of nodes and number of packets per second.

Due to the less traffic congestion and immediate route identification from $\mathrm{CH}$ to the AUV, throughput for transmitted packets is high. Unfortunately, existing works have obtained high communication overhead and high traffic congestion. When network size is expands then the performance of throughput is low. Hence, ACMC is not able to handle the high volume of traffic. ESRVR is suited only 
when the network has limited number of nodes and processing with limited number of communications. Since, it uses two-hop information for routing packets. This is insufficient in achieving higher throughput. A large number of void holes decrease the network performance. In this case, frequent void hole mitigation is important that degrades network throughput.
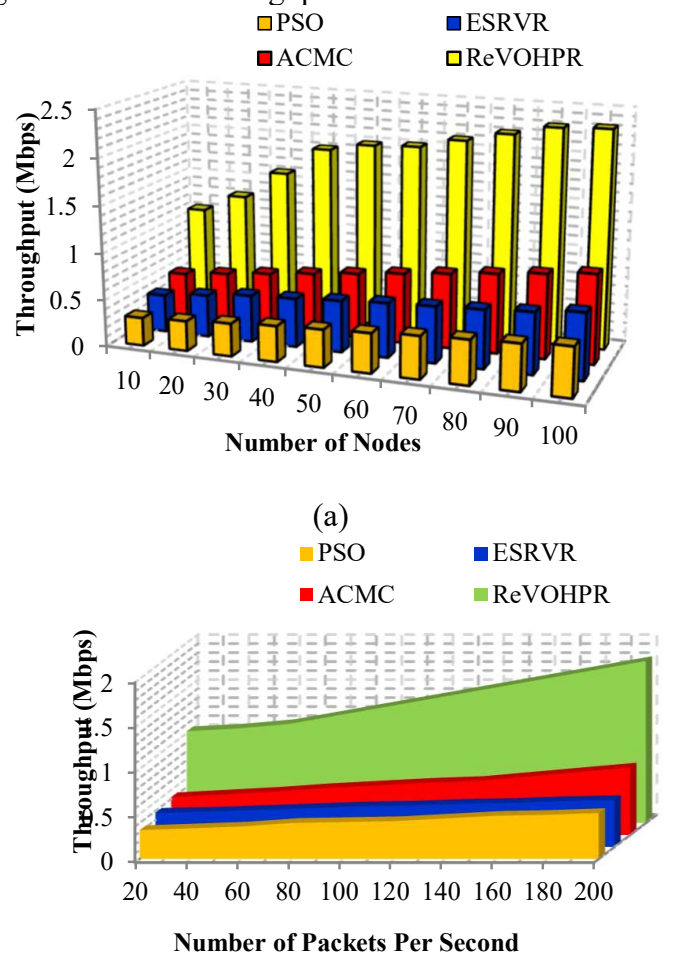

(b)

Fig.13.Impact of Throughput

Fig 13 shows the performance throughput with respect the number of packets processed per second. Relay based void hole detection and mitigation addresses and avoids the multi-void holes prediction. This problem improves energy efficiency and QoS based metrics such as throughput, delay and PDR. In ACMC, clusters are formed using K-means algorithm in which centroid selection must be optimum and cluster size must be known. Data transfer time and mode is frequently changed i.e. adaptive in the proposed protocol. Hence, we obtained higher throughput than the previous protocols. We have used the end-to end approach for improving network throughput for a longer period.

\section{d. PDR}

PDR is the packet delivery rate metric that analyzed for every node in the network in data transmission time. It is defined as the sum of packets successfully received at the destination node from the source node. It is computed by follows,

$$
P D R=\frac{N(P(R))}{N(P(G))}
$$

Where $N(P(R))$ is the number of packets received at the source node $N(P(G))$ is the number of packets generated at the source node.

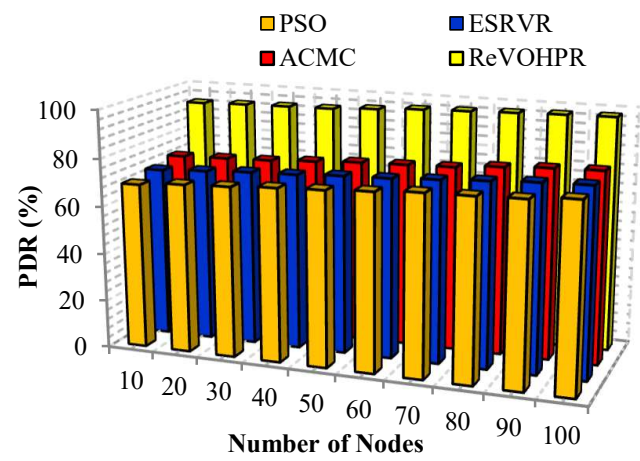

(a)

$$
\begin{array}{ll}
\square \text { PSO } & \square \text { ESRVR } \\
\square \text { ACMC } & \text { ReVOHPR }
\end{array}
$$

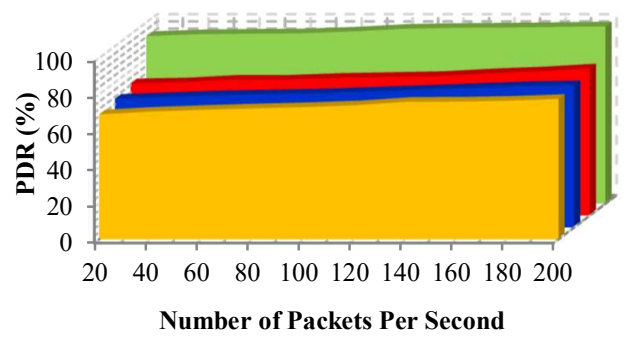

(b)

Fig.14.Impact of PDR

Fig 14 shows the simulation results for the PDR with respect to the number of nodes and number of packets processed per second. The plot of PDR in ReVOHPR increases with network density increases. Optimum relay selection for routing packets from source $\mathrm{CH}$ to the destination $\mathrm{CH}$ improves the PDR and also number of void holes are detected and mitigated in the proposed protocol. The existing protocol i.e. ACMC uses simple void handling procedure that failed since single metric is considered for void hole detection. On behalf of void hole mitigation, packets transmitted to the next hop is guaranteed and also it ensures the packet delivery. When void hole is determined, then relay node that near to the In contrast, a previous protocol such as PSO and ESRVR does not fit for robust data transmission. In PSO, two level CHs are elected which increases overhead in data transmission. Further, PSO, void hole prediction consumes more processing and hence packet losses are very high whereas ESRVR uses two hops for data transmission. The selection for two hops is not reliable in this work. Hence, PDR is very lower.

\section{Results and Discussion}

Based on the simulation results of the proposed REVOHPR protocol with the previous protocols, it is concluded that the REVOHPR offers various benefits. Due to the development new protocol for data transmission and void hole mitigation in UWSN, the number of active nodes rate is increases, where the dead nodes count is reduced. The minimization of energy consumption and the improvement in overall QoS in the proposed REVOHPR is due to the following set of ideas implemented.

- Cluster formation and optimum $\mathrm{CH}$ selection by E2R protocol improves these processes and avoids 
frequent cluster formation by considering the centrality factor and success rate of each node. By this method $37 \%$ of energy consumption is reduced.

- Void hole repair algorithm addresses the packet dropping issues and also eliminates the packet retransmission.

- Virtual graph construction process reduces the complexity by $50 \%$, which increases the lifetime of network by half than the previous protocols.

\section{CONCLUSION}

In this paper, void hole problem is addressed for energy consumption reduction. For this purpose, ReVOHPR protocol is proposed which deals with the four processes and sensors are heterogeneous in nature and they are level based clustering in which E2R protocol is presented for stable $\mathrm{CH}$ election. Then dynamic sleep scheduling mechanism is considering to improve the lifetime of a network which is dynamic by implementing the DK2F algorithm. Virtual graph based routing algorithm is presented for data transmission in which virtual route is established between the source $\mathrm{CH}$ to the destination. To avoid data transmission delay, multiple AUVs deployed to gather data packets. Finally, relay assisted void hole detection and repair is presented which eliminates the multiple void hole problems for a longer period. Our simulation results show that the proposed ReVOHPR protocol exceeds the performance than baseline protocols as ESRVR, ACMC and PSO in terms of energy consumption, packet delivery ratio, throughput, and delay.

In future, we planned to focus on the security aspect of data transmission to avoid threats in UWSN. In this case, various attacks in UWSN is detected and mitigated to further reduce the energy consumption and improve the QoS. Future work avenues include the investigation of more efficient network coverage based on UAV $[39,40]$.

\section{REFERENCES}

[1] Yang, G., Dai, L., Si, G., Wang, S., \& Wang, S. (2018). Challenges and Security Issues in Underwater Wireless Sensor Networks. IIKI.

[2] Kanthimathi, N., \& Dejey (2017). Void handling using Geo-Opportunistic Routing in underwater wireless sensor networks. Comput. Electr. Eng., 64, 365-379.

[3] Coutinho, R.W., Boukerche, A., Vieira, L.F., \& Loureiro, A. (2017). Performance modeling and analysis of void-handling methodologies in underwater wireless sensor networks. Comput. Networks, 126, 114.

[4] Ahmed, M., Salleh, M., \& Channa, M. I. (2018). Routing protocols based on protocol operations for underwater wireless sensor network: A survey. Egyptian Informatics Journal, 19(1), 57-62.

[5] Shakila, R., \& Paramasivan, B. (2020). Performance Analysis of Submarine Detection in Underwater Wireless Sensor Networks for Naval Application. Microprocessors and Microsystems, 103293.

[6] Wang, D., Liu, J., \& Yao, D. (2020). An Energyefficient Distributed Adaptive Cooperative Routing Based on Reinforcement Learning in Wireless Multimedia Sensor Networks. Computer Networks, 107313.

[7] Jouhari, M., Ibrahimi, K., Tembine, H., \& Ben-Othman, J. (2019). Underwater Wireless Sensor Networks: A survey on enabling technologies, localization protocols, and Internet of Underwater Things. IEEE Access, 1-1.
[8] Basagni, S., Valerio, V. D., Gjanci, P., \& Petrioli, C. (2018). MARLIN-Q: Multi-Modal Communications for Reliable and Low-latency Underwater Data Delivery. Ad Hoc Networks.

[9] Ismat, N., Qureshi, R., Enam, R. N., Noor, S., \& Tahir M. (2020). Cluster Estimation in Terrestrial and Underwater Sensor Networks. Wireless Personal Communications.

[10] Zhang, M., \& Cai, W. (2020). Energy-efficient Depth based Probabilistic Routing within 2-Hop Neighborhood for Underwater Sensor Networks. IEEE Sensors Letters, $1-1$.

[11] Ahmed, M., Salleh, M., \& Channa, M. I. (2016). Routing protocols for underwater wireless sensor networks based on data forwarding: a review. Telecommunication Systems, 65(1), 139-153.

[12] Wang, Z., Han, G., Qin, H., Zhang, S., \& Sui, Y. (2018). An Energy-Aware and Void-Avoidable Routing Protocol for Underwater Sensor Networks. IEEE Access, 6, 7792-7801.

[13] Ahmed, M., Salleh, M., \& Channa, M.I. (2018). CBE2R: clustered-based energy efficient routing protocol for underwater wireless sensor network. International Journal of Electronics, 105, 1916 $-1930$.

[14] Chen, M., \& Zhu, D. (2019). Data collection from underwater acoustic sensor networks based on optimization algorithms. Computing.

[15] Jamshidi, A. (2018). Efficient cooperative ARQ protocols based on relay selection in underwater acoustic communication sensor networks. Wireless Networks.

[16] Javaid, N., Ahmed, F., Wadud, Z., Alrajeh, N., Alabed, M., \& Ilahi, M. (2017). Two Hop Adaptive Vector Based Quality Forwarding for Void Hole Avoidance in Underwater WSNs. Sensors (Basel, Switzerland), 17.

[17] Javaid, N., Karim, O., Sher, A., Imran, M., Yasar, A., \& Guizani, M. (2018). Q-Learning for energy balancing and avoiding the void hole routing protocol in underwater sensor networks. 2018 14th International Wireless Communications \& Mobile Computing Conference (IWCMC), 702-706.

[18] Latif, G., Javaid, N., Sher, A., Khan, M., Hameed, T., \& Abbas, W. (2018). An Efficient Routing Algorithm for Void Hole Avoidance in Underwater Wireless Sensor Networks. 2018 IEEE 32nd International Conference on Advanced Information Networking and Applications (AINA), 305-310.

[19] Sher, A., Khan, A., Javaid, N., Ahmed, S.H., Aalsalem, M.Y., \& Khan, W. (2018). Void Hole Avoidance for Reliable Data Delivery in IoT Enabled Underwater Wireless Sensor Networks. Sensors (Basel, Switzerland), 18

[20] Signori, A., Campagnaro, F., Steinmetz, F., Renner, C., \& Zorzi, M. (2019). Data Gathering from a Multimodal Dense Underwater Acoustic Sensor Network Deployed in Shallow Fresh Water Scenarios. J. Sens. Actuator Networks, 8, 55.

[21] Banaeizadeh, F., \& Haghighat, A.T. (2020). An energyefficient data gathering scheme in underwater wireless sensor networks using a mobile sink. International Journal of Information Technology, 12, 513-522.

[22] Kumari, S., Mishra, P.K., \& Anand, V. (2020). Fault resilient routing based on moth flame optimization scheme for underwater wireless sensor networks. Wirel. Networks, 26, 1417-1431.

[23] Ghoreyshi, S.M., Shahrabi, A., Boutaleb, T., \& Khalily, M. (2019). Mobile Data Gathering With HopConstrained Clustering in Underwater Sensor Networks. IEEE Access, 7, 21118-21132.

[24] Khan, M.R., Ahmed, S.H., \& Kim, D. (2019). AUVAided Energy-Efficient Clustering in the Internet of Underwater Things. IEEE Transactions on Green Communications and Networking, 3, 1132-1141. 
[25] Zhang, W., Wang, J., Han, G., Zhang, X., \& Feng, Y. (2019). A Cluster Sleep-Wake Scheduling Algorithm Based on 3D Topology Control in Underwater Sensor Networks. Sensors (Basel, Switzerland), 19.

[26] Islam, T., \& Park, S. (2020). A Two-Stage Routing Protocol for Partitioned Underwater Wireless Sensor Networks. Symmetry, 12, 783.

[27] Wadud, Z., Ismail, M.A., Qazi, A.B., Khan, F.A., Derhab, A., Ahmad, I., \& Ahmad, A.M. (2019). An Energy Balanced Efficient and Reliable Routing Protocol for Underwater Wireless Sensor Networks. IEEE Access, 7, 175980-175999.

[28] Guan, Q., Ji, F., Liu, Y., Yu, H., \& Chen, W. (2019). Distance-Vector-Based Opportunistic Routing for Underwater Acoustic Sensor Networks. IEEE Internet of Things Journal, 6, 3831-3839.

[29] Akbar, M., Javaid, N., Khan, A., Imran, M., Shoaib, M., \& Vasilakos, A. (2016). Efficient Data Gathering in 3D Linear Underwater Wireless Sensor Networks Using Sink Mobility. Sensors (Basel, Switzerland), 16.

[30] Faheem, M., Ngadi, M. A., \& Gungor, V. C. (2019). Energy Efficient Multi-Objective Evolutionary Routing Scheme for Reliable Data Gathering in Internet of Underwater Acoustic Sensor Networks. Ad Hoc Networks, 101912.

[31] Krishnaswamy, V., \& Manvi, S. (2019). Fuzzy and PSO Based Clustering Scheme in Underwater Acoustic Sensor Networks Using Energy and Distance Parameters. Wireless Personal Communications, 1-18.

[32] Ateya, A. A., Muthanna, A., Vybornova, A., Algarni, A. D., Abuarqoub, A., Koucheryavy, Y., \& Koucheryavy, A. (2019). Chaotic salp swarm algorithm for SDN multi-controller networks. Engineering Science and Technology, an International Journal, 22(4), 1001-1012. https://doi.org/10.1016/j.jestch.2018.12.015

[33] Awais, M., Ali, I., Alghamdi, T., Ramzan, M., Tahir, M., Akbar, M., \& Javaid, N. (2020). Towards Void Hole Alleviation: Enhanced GEographic and Opportunistic Routing Protocols in Harsh Underwater WSNs. IEEE Access, 8, 96592-96605.

[34] Khan, A.A., Aurangzeb, K., Qazi, E., \& Rahman, A.U. (2019). Energy-Aware Scalable Reliable and Void-Hole Mitigation Routing for Sparsely Deployed Underwater Acoustic Networks. Applied Sciences, 10, 177.

[35] Muthanna M.S.A., Wang P., Wei M., Ateya A.A., Muthanna A. Toward an ultra-low latency and energy efficient LORAWAN Internet of Things, Smart Spaces, and Next Generation Networks and Systems 2019. C. 233-242.

[36] Wang, Q., Li, J., Qi, Q., Zhou, P., \& Wu, D.O. (2020). A Game Theoretic Routing Protocol for 3D Underwater Acoustic Sensor Networks. IEEE Internet of Things Journal, 1-1.

[37] Huang, M., Zhang, K., Zeng, Z., Wang, T., \& Liu, Y. (2020). An AUV-assisted Data Gathering Scheme based on Clustering and Matrix Completion for Smart Ocean. IEEE Internet of Things Journal, 1-1.

[38] Jin, Z., Zhao, Q., \& Luo, Y. (2020). Routing Void Prediction and Repairing in AUV-Assisted Underwater Acoustic Sensor Networks. IEEE Access, 8, 5420054212.

[39] Ateya, A. A. A., Muthanna, A., Kirichek, R., Hammoudeh, M., \& Koucheryavy, A. (2019). Energyand Latency-Aware Hybrid Offloading Algorithm for UAVs. IEEE Access, 7, 37587-37600. https://doi.org/10.1109/ACCESS.2019.2905249

[40] Ateya, Abdelhamied A., Ammar Muthanna, Irina Gudkova, Yuliya Gaidamaka, and Abeer D. Algarni. 2019. "Latency and Energy-Efficient Multi-Hop Routing Protocol for Unmanned Aerial Vehicle Networks." International Journal of Distributed Sensor
Networks 15 (8). SAGE Publications Ltd. doi: $10.1177 / 1550147719866392$. 